\title{
Pregnancy: Effect on Multiple Sclerosis, Treatment Considerations, and Breastfeeding
}

\author{
Rhonda Voskuhl $^{1}$ • Callene Momtazee ${ }^{1}$
}

Published online: 1 August 2017

(C) The American Society for Experimental NeuroTherapeutics, Inc. 2017

\begin{abstract}
Multiple sclerosis (MS) commonly affects women in childbearing years making pregnancy issues important for patients with MS and their families. Pregnancy is a naturally occurring disease modifier of MS associated with a $70 \%$ reduction in relapse rates in the third trimester. This relapse rate reduction during the last trimester is roughly equal to the most effective disease-modifying treatments for MS. Given this efficacy, various pregnancy factors have been tested to determine which play a part in pregnancy's protection, and some have been translated to completed and ongoing phase II clinical trials. In contrast to protective effects during pregnancy, the postpartum period entails increased relapse risk, which may be due to either abrupt removal of protective pregnancy factors after delivery or to unique deleterious factors inherent to the postpartum period. The effect of breastfeeding on MS remains unclear. The best predictor for whether a patient will have a postpartum relapse is the incidence of her having active relapsing MS prior to pregnancy. The medical management of MS during pregnancy and the postpartum period is challenging given the risks of medication exposure to the fetus in utero and to the infant through breast milk. This review will focus on clinical aspects of pregnancy, including the effects of pregnancy on MS disease activity, as well as the medical management of MS during pregnancy and postpartum.
\end{abstract}

Electronic supplementary material The online version of this article (doi:10.1007/s13311-017-0562-7) contains supplementary material, which is available to authorized users.

Rhonda Voskuhl

rvoskuhl@mednet.ucla.edu

1 Multiple Sclerosis Program, UCLA Department of Neurology, David Geffen School of Medicine, University of Los Angeles, Los Angeles, CA 90095, USA
Keywords Multiple sclerosis · Pregnancy · Estrogen · Estriol $\cdot$ Breastfeeding $\cdot$ Disease-modifying therapies

\section{Effect of Pregnancy on Multiple Sclerosis}

Multiple sclerosis (MS) incidence is 2 to 3 times higher in women than men. MS onset is common in women aged 20 to 40 years [1,2]; as these are childbearing years, the effect of pregnancy on MS is an important clinical issue.

Pregnancy has a beneficial effect in MS and its most widely used animal model, experimental autoimmune encephalomyelitis (EAE). Protection from EAE during pregnancy has been observed across a range of species [3]. Therefore, this protection is thought to be due to basic biologic mechanisms and is not limited to subjective perspectives or social effects of pregnancy in patients with MS. Immunomodulation of the maternal immune system is known to occur naturally and has long been thought to be protective for the developing fetus. A fetus is a half foreign allograft since it contains paternal proteins. Indeed, other cell-mediated autoimmune diseases also ameliorate during pregnancy, including psoriasis and rheumatoid arthritis [2]. Many changes occur during pregnancy, and any could be responsible for this immunomodulation, including an increase in estrogens (estradiol, estriol) and progesterone, to name a few. These hormones increase gradually during pregnancy and peak in the third trimester, the time of greatest disease protection. Other hormones such as cortisol also change, albeit to a lesser degree, during pregnancy. Additional molecules change very early after conception and are short lived, making them less likely to contribute to disease protection in the last trimester. Estrogens not only reach highest levels late in pregnancy, but have also been shown to be neuroprotective in several neurological disease models [4-7], and are known to play a role in normal cognitive 
development $[8,9]$. We hypothesize that it is evolutionarily advantageous for a pregnancy factor to be both immunomodulatory and neuroprotective, because these properties increase the survival of the fetus as an allograft and protect the developing brain from injury during maternal insults. While this elegant mechanism may exist to protect the fetus, it is also ideal for mothers with MS.

\section{Effect of Pregnancy on MS Relapses}

For decades, it was anecdotally observed that women with MS appeared to have fewer relapses during pregnancy, but then had increased relapses postpartum. Further clinical observations noted that the onset of MS occurred in some patients during the postpartum period. In a landmark paper [10], 227 patients with MS were followed prospectively and showed an approximate $70 \%$ reduction in relapse rates during the third trimester of pregnancy. In this same study, the postpartum relapse rate was higher than before pregnancy. Over the following postpartum year, this temporary increase in relapses subsided, and rates returned to baseline prepregnancy levels.

\section{Other Effects of Pregnancy on MS}

Whether the temporary protection from relapses during the late pregnancy and the temporary increase in relapses postpartum has a net effect on permanent disability accumulation has been controversial [11-13]. Short-term studies following patients for 2 years or less showed no net effect of a single pregnancy on disability level. However, longer-term studies following patients for many years suggested that women with multiple pregnancies have less disability and/or took more time to reach a given disability level [14-16]. In a cohort of 2466 patients followed for up to 10 years, a higher number of pregnancies were independently associated with lower disability scores [17]. However, this could have been due, in part, to a selection bias whereby women with less disabling MS were more likely to become pregnant multiple times. Based on the known effect of anti-inflammatory disease-modifying therapies (DMTs) in MS, where several years of continuous treatment modestly slows disability worsening, the worsening of disability would be unlikely given that temporary immunomodulation during the second half of pregnancy could result in permanent effects on disability. However, it is possible that repeated exposure to neuroprotective pregnancy hormones at high levels during the third trimester may impact rates of disability accumulation. This area warrants further study.

Another potential effect of pregnancy is that being multiparous, versus nulliparous, decreases a women's likelihood of developing MS. An Australian study of 282 patients with MS and 542 controls found that having increased numbers of pregnancies was associated with a lower risk of developing the first demyelinating event [18]. The effect of pregnancy decreased the risk with an odds ratio of $50 \%$ per pregnancy. This was an elegant study because focus on the first demyelinating event, instead of a relapse in established MS, permitted distinction between the biological effect of pregnancy on disease versus the potential bias that having disease may exert on the patient's decision to become pregnant. This study prompted speculation that the trend for women over the last several decades to delay their first pregnancy and have fewer children may contribute to the known increasing incidence of MS in females. Specifically, the female to male sex difference in MS incidence has increased from approximately 2:1 to 3:1 [19]. In summary, there is no proof that pregnancy can protect a genetically susceptible patient from developing MS. However, women with a family history of MS should be reassured that having multiple pregnancies will not negatively impact their risk of developing MS [20].

\section{Clinical Trials Aiming to Simulate the Protection of Pregnancy in MS}

The strongest clinical observation to date of a naturally occurring disease modifier in MS is the $70 \%$ reduction in relapses in the third trimester of pregnancy. Preclinical studies showed that the pregnancy estrogen, estriol, was protective in EAE when used at a dose physiologic with levels during pregnancy [21-26]. Translation to clinical trials followed. Estriol treatment using an oral dose of $8 \mathrm{mg}$ per day to induce a pregnancy level in the blood of nonpregnant MS women was studied in phase II clinical trials. This rapid translation to the clinic was possible because of the pre-existing safety track record of estriol as one of the safest of estrogens [27-30], thereby circumventing the need for phase I toxicity studies. A pilot, single-arm, crossover phase IIa trial of estriol treatment showed an $80 \%$ reduction in gadolinium-enhancing lesions as compared with pretreatment on serial monthly brain magnetic resonance imaging (MRI) as the primary outcome measure [31]. Cessation of treatment showed an increase in enhancing lesion activity, and resumption of treatment showed another reduction in enhancing lesions. In addition, antiinflammatory effects in immune cells in the blood were shown $[32,33]$.

Next, a double-blinded, placebo-controlled phase IIb trial of estriol treatment was done with the clinical outcome of relapse rate as the primary outcome measure [34]. This was unusual since phase II trials in MS have used the biomarker of MRI-enhancing lesions as the primary outcome. However, phase II trials in several other diseases have used a clinical outcome, instead of a biomarker, as the primary outcome measure, thereby creating essentially an underpowered phase III trial. The relatively smaller sample size of a phase II trial compared with a phase III trial required a $p$-value of 0.10 , 
versus a $p$-value of 0.05 , which was the case with the estriol phase IIb trial as well $[34,35]$. The estriol trial in MS was an add-on study of estriol versus placebo, given in combination with standard of care treatment with glatiramer acetate (GA). In total, 158 patients with relapsing-remitting MS (RRMS) were randomized (1:1) to receive GA plus placebo or GA plus estriol for 24 months. Estriol treatment significantly reduced annualized relapse rates as the primary outcome measure and increased the time to first relapse as the secondary outcome, as prespecified. In addition, there were promising effects on exploratory outcomes, namely reducing fatigue, increasing performance on cognitive testing, and sparing cortical gray matter atrophy, each as compared with placebo. Poor compliance late in the trial resulted in significantly lower estriol levels at month 24 , which coincided with the loss of effects on cognition and gray matter sparing during month 24 evaluations. Indeed, across the entire trial duration, higher estriol blood levels correlated with better cognition, and better cognition was correlated with cortical gray matter sparing. To follow up on these promising effects on cognition, another phase $\mathrm{IIb}$, double-blinded, placebo-controlled trial of estriol treatment is ongoing with improvement in cognitive testing as the primary outcome measure (NCT01466114).

Regarding other pregnancy factors that could mediate disease protection during the last trimester of pregnancy, another trial treated patients with estradiol [36]. Synthetic estradiol in oral contraceptives was used at doses to induce blood estradiol levels physiologic with levels of the ovarian cycle. Higher doses of estradiol to mimic blood levels in the third trimester of pregnancy would not be well tolerated given the potential for deleterious effects due to the high affinity of estradiol for estrogen receptor alpha in breast and uterine tissue. MS women received either $40 \mathrm{mg}$ or $20 \mathrm{mg}$ ethinylestradiol, or placebo. This was an add-on study to standard-of-care treatment with interferon (IFN)- $\beta 1 \mathrm{a}$. In total, 150 patients with RRMS were randomized (1:1:1) to receive IFN- $\beta 1$ a only or IFN- $\beta 1$ a with low-dose $(20 \mathrm{mg})$ ethinylestradiol plus desogestrel or IFN- $\beta 1 \mathrm{a}$ and high-dose $(40 \mathrm{mg})$ ethinylestradiol plus desogestrel for 96 weeks. The level of cumulative active inflammatory lesions on brain MRI was reduced by $26 \%$ with the 40-mg ethinylestradiol dose versus placebo, with the 20$\mathrm{mg}$ dose having an intermediate, but not significant, reduction. However, this MRI outcome was not a prespecified primary endpoint, and there was no benefit on relapse rate or disability, the 2 primary endpoints. Another exploratory finding in the estradiol-containing oral contraceptive pill (OCP) trial was that the 40-mg dose was associated with better cognitive performance versus placebo, whereas the 20-mg dose was not [37]. Together these data reinforce the importance of estrogen type and dose in modifying MS.

Progesterone also increases gradually during pregnancy, reaching highest levels in the last trimester. Estrogens and progesterone drop precipitously after delivery. Along with this drop in female hormones, an increase in MS relapses is seen postpartum. Thus, a clinical trial of a synthetic progesterone treatment was undertaken to prevent postpartum relapses [38]. The choice of progesterone was based, in part, on preclinical data of well-characterized beneficial effects of progesterone on myelination $[39,40]$. The trial was stopped early owing to futility. In hindsight, this was not surprising given progesterone's inconsistent effects in significantly ameliorating disease in the MS preclinical model EAE [41-44]. Perhaps future trials could be designed to assess whether progesterone treatment improves remyelination as the primary outcome measure, since this would be more aligned with known progesterone effects rather than assessment of its effect on relapses.

While estrogens and progesterone play significant roles in pregnancy, they are not the only physiologic changes a woman under goes during gestation. Whereas the aforementioned clinical trials using estrogens show promise as being the principal factors responsible for the beneficial effects of pregnancy on MS, there are other factors that warrant further study. Indeed, while estriol is the most advanced in clinical trials, this does not rule out additional effects from other factors increased during pregnancy, including corticosteroids and vitamin D, particularly since synergism between these factors with estrogens has been reported [45-50].

\section{Management of MS Medications During Pregnancy}

Ideally, clinicians and their patients with MS should discuss family planning as early as possible in the course of treatment. This way, patients may make informed decisions about their medication choices during pregnancy while maintaining optimal disease protection throughout pregnancy and the postpartum period. Some patients do not consider pregnancy as a possibility or realize that they may need to stop medications several months prior to trying to conceive. By addressing these concerns early with a complement of medical professionals (including neurologists, maternal fetal medicine specialists, and lactation specialists among others), medical care of the whole woman can be optimized.

\section{Fertility and MS}

Patients with MS are not less fertile and do not have more difficulty in completing a pregnancy to term compared with healthy controls. That said, as in the general population, some patients with MS will experience infertility and wish to undergo treatment. The overall risk of infertility for couples in Western nations is roughly $10 \%$ to $20 \%$. A recent prospective study by Correale et al. [51] in patients with MS aimed to identify what risks, if any, are seen with assisted reproductive technology (ART). 16 patients with MS undergoing ART were followed prospectively 12 months before and 9 months 
after their final cycle of ART. An increase in MS exacerbations and MRI lesion activity was seen compared with matched patients with MS not undergoing ART. Based upon this study, as well as other observational papers in ART, it is recommended that patients undergoing ART be cautioned about these risks and watched carefully for intervention as necessary. MS DMTs themselves, rather than the disease of MS, may affect a patient's ability to conceive or have a deleterious effect on the fetus. For example, traditional chemotherapy agents like cyclophosphamide may affect gamete production or viability. Hence, sperm and egg banking can be put forth as options for male and female patients if future children are desired after these treatments. Each DMT has different potential effects on the fetus, and they vary in the lengths of time they should be discontinued prior to any attempt to conceive. In this regard, the US Food and Drug Administration (FDA) classification system is helpful in identifying medications that may be more or less suitable for women (and men) with MS of childbearing age.

\section{FDA Classifications}

Clinicians typically turn to the FDA designations listed below to assess what the overall risk for a particular drug may pose to their patients who are pregnant or lactating: A) no evidence of fetal harm in human studies; B) no evidence of fetal harm in animal studies; C) evidence of fetal harm in animal studies or no data available; D) evidence of fetal harm in humans; use may be justified in some circumstances; $X$ ) evidence of fetal harm in humans; not indicated for use in pregnancy

However, these safety categories during pregnancy are currently being revised by the FDA. During this transitional period, there are 2 FDA classifications being used by physicians. The above FDA pregnancy risk letter designations are still in use for prescription labeling for medications introduced prior to June 30, 2015.

The newer drug labeling, currently being formulated, will summarize data concerning the known risks and benefits of a particular medication that is systemically absorbed during pregnancy and lactation, as well as any fertility implications for both women and men. Older medications will begin phasing in the use of the new narrative designations of risk for use in pregnancy and lactation soon.

The current FDA-approved DMTs and commonly used symptom management medications in MS are listed in Tables 1 and 2 respectively, along with their current pregnancy letter safety categories or narrative descriptions.

\section{Contraceptive and Family Planning Issues}

Roughly half of all pregnancies in the USA are unintended [52]. There are no hard data on the number of unplanned pregnancies specifically in patients with MS. It is possible that the MS population would be more likely to make a concerted effort in family planning given the impact the disease has on their social and economic futures, as well as the fact that they are on medications that can sometimes affect fertility or be teratogenic. Thus, clinicians may make an argument for using types of birth control that are most effective at preventing pregnancy in order to give patients as much control over their reproductive futures as possible. According to the Centers for Disease Control, the most effective types of birth control include implants or an intrauterine device, both of which result in $<1$ pregnancy per 100 women in a year. This is considerably better than the oral contraceptive pill, which has a failure rate of up to $9 \%$ for typical use, and the male condom at $18 \%$.

Overall, any type of contraception is safe for patients with MS and it is up to the patient to choose their preferred method with their primary care doctor or gynecologist. But many patients will ask if one type of contraception is preferable to another. Unfortunately, clinical data do not support a definitive choice. The dose and type of estrogen appear to influence outcomes in clinical trials in MS. Estradiol, or a synthetic variant of it, is the estrogen often in oral contraceptives, but some contain no estrogens and only progestins. Retrospective data assessing the use of OCPs did not show a beneficial effect in preventing subsequent development of MS; however, recent or current use showed some potential benefit [53-55]. Unfortunately, these studies grouped together various doses of estrogen containing OCPs, as well as progesterone-only OCPs, each taken for different durations per month. There is no evidence that low-dose estrogen containing OCPs would affect MS disease activity. Also, there is no current evidence that progesterone-only-containing OCPs or progesteronecontaining intrauterine devices would affect MS.

For patients with MS hoping to become pregnant, a planning visit should be scheduled at least 6 months in advance of efforts to conceive, to discuss when and if MS-related medications should be stopped. Some drugs are relatively safe for use during pregnancy, and others may need to be discontinued up to 6 months to 2 years prior to conception. Advice must be tailored to each patient based on risk of treatment-related adverse effects on pregnancy versus risk of MS relapses during the untreated period when the patient is trying to conceive.

\section{The Use of DMTs During Pregnancy}

In the past, it was generally advised that no DMTs for MS be taken during pregnancy as none was approved for use. Even today, there are no prospective studies or clear guidelines on the use of DMTs during pregnancy and lactation. However, there is some evidence from ongoing pregnancy registries that certain DMTs are relatively safer for the developing fetus. This may aid in decision-making for patients who either have a high level of MS disease activity or are having difficulty 
Table 1 Pregnancy safety categories for multiple sclerosis (MS) disease-modifying therapies

\begin{tabular}{|c|c|}
\hline $\begin{array}{l}\text { FDA-approved MS } \\
\text { therapies }\end{array}$ & Pregnancy category \\
\hline $\mathrm{IFN}-\beta \mathrm{Ib}$ & $\mathrm{C}$ \\
\hline IFN- $\beta$ Ia & $\mathrm{C}$ \\
\hline Fingolimod & $\mathrm{C}$ \\
\hline Glatiramer acetate & $\mathrm{B}$ \\
\hline Dimethyl fumarate & $\mathrm{C}$ \\
\hline Teriflunomide & $\mathrm{X}$ \\
\hline Alemtuzumab & $\mathrm{C}$ \\
\hline Daclizumab & $\begin{array}{l}\text { Narrative: no adequate data on risk in pregnant women, but animal data showed } \\
\text { embryofetal death and reduced growth at exposures of } 30 \times \text { clinical usages }\end{array}$ \\
\hline Natalizumab & $\mathrm{C}$ \\
\hline Ocrelizumab & $\begin{array}{l}\text { Narrative: no adequate data on risk in pregnant women, B-cell depletion and } \\
\text { lymphocytopenia of a transient nature in infants of mothers treated with } \\
\text { anti-CD20 antibodies during pregnancy }\end{array}$ \\
\hline Mitoxantrone & $\mathrm{D}$ \\
\hline $\begin{array}{l}\text { Non-FDA approved MS } \\
\text { therapies }\end{array}$ & \\
\hline Azathioprine & $\mathrm{D}$ \\
\hline Cyclophosphamide & $\mathrm{D}$ \\
\hline IVIG & $\mathrm{C}$ \\
\hline Rituximab & $\mathrm{C}$ \\
\hline Methotrexate & $\mathrm{X}$ \\
\hline Methylprednisolone & $\mathrm{C}$ \\
\hline Mycophenolate mofitil & $\mathrm{D}$ \\
\hline
\end{tabular}

FDA = Food and Drug Administration; IFN = interferon; IVIG = intravenous immunoglobulin getting pregnant and do not wish to be off DMTs for a prolonged period of time.

In a particular study, GA was used by patients throughout pregnancy and no increase in malformations or miscarriages was seen compared with the normal population [56]. Interestingly, a low incidence of MS relapses was observed in the postpartum period. However, it is not clear whether this possible protection postpartum was due to continuation of GA treatment during pregnancy or due to a selection bias whereby

Table 2 Food and Drug Administration pregnancy safety categories for symptom-management therapies

\begin{tabular}{lll}
\hline Agent & Symptom & Pregnancy risk category \\
\hline Corticosteroid & Acute exacerbation & $\mathrm{C}$ \\
Baclofen & Spasticity & $\mathrm{C}$ \\
Diazepam & Spasticity, anxiety & $\mathrm{D}$ \\
Tizanidine & Spasticity & $\mathrm{C}$ \\
Gabapentin & Seizure, pain, spasticity & $\mathrm{C}$ \\
Amantadine & Fatigue & $\mathrm{C}$ \\
Modafinil & Fatigue & $\mathrm{C}$ \\
Oxybutinin & Overactive bladder & $\mathrm{B}$ \\
Tolterodine & Overactive bladder & $\mathrm{C}$ \\
Dalfampridine & Improved walking speed & $\mathrm{C}$ \\
\hline
\end{tabular}

GA-treated women had relatively milder disease prior to pregnancy. Also, in a recent registry of pregnancies exposed to GA, no increase in teratogenicity or miscarriage was seen. Based upon these studies and many others, GA is now approved in the European Union for use during pregnancy in patients with RRMS.

IFN- $\beta$ has shown no teratogenicity in animal studies, but there was a minimal increase in miscarriage rate with supratherapeutic human doses, which led to its category $\mathrm{C}$ rating by the FDA. In a registry of IFN- $\beta$ users as part of the German Multiple Sclerosis and Pregnancy Registry, there were 251 IFN- $\beta$-exposed pregnancies that showed no increase in preterm birth, spontaneous abortion, or congenital abnormalities compared with pregnancies unexposed to treatment [57]. Additionally, the Betaseron pregnancy registry of 99 patients with MS who used IFN- $\beta$ almost exclusively in the first trimester did not show any increase in miscarriage or birth defects [58]. This is similar to the results of the Avonex Pregnancy Exposure Registry (IFN- $\beta 1 \mathrm{a}, n=306$ ). Based upon these long-term results, it is now considered safe to continue IFN until conception or stop 1 cycle prior if an abundance of caution is desired.

Fingolimod has a known association with cardiovascular malformations and increased spontaneous abortions in pregnant animals and is classified as category C. During the 
preclinical trials using fingolimod, 66 pregnancies were identified with in utero exposure, but no firm conclusions could be drawn owing to small sample size. An official pregnancy registry for fingolimod is ongoing [59].

A monitoring study of almost 200 pregnancies with exposure to dimethyl fumarate from preclinical and postmarketing settings showed no increase in adverse pregnancy outcomes or fetal risks compared with the general population [60].

Interestingly, teriflunomide, which is a category Xdesignated DMT based upon its known teratogenicity in animals, had a pregnancy and paternal exposure series that showed limited risks, if any, to the developing human fetus. Teriflunomide is also unique in that it is found in human semen in small amounts, but it is not known to damage sperm or affect male fertility. In this retrospective analysis, 83 pregnancies in females and 22 pregnancies that were the result of teriflunomide-exposed male patients were identified. Only 26 of the female pregnancies were carried to term and exposed to teriflunomide and 19 of the male exposed pregnancies. The bulk of these patients used the accelerated elimination procedure to decrease teriflunomide exposure once pregnancy was identified. None of the drug-exposed infants had any significant congenital abnormalities and no increased spontaneous abortions were seen [61]. The remaining pregnancies resulted in induced abortions. Despite these encouraging findings for patients who become pregnant on teriflunomide, the strong recommendation remains to use consistent and effective birth control while on this drug to prevent pregnancy and offer accelerated elimination of teriflunomide if conception is desired or an unintended pregnancy arises.

Results from the Tysabri Pregnancy Exposure Registry, which enrolled 369 patients with MS and 7 patients with Crohn's disease, demonstrated a slightly elevated risk of fetal malformations of $5.05 \%$ verus the $2.67 \%$ standard reference rate for the general population. As no consistent pattern of congenital defects was seen, no definitive drug effect was concluded. Most of the patients in this registry discontinued study drug during the first trimester, and the remainder generally within 3 months prior to conception. Relatively few studied continued the drug into the second and third trimesters. Natalizumab has been shown in animal studies to cross the placenta and was associated with hematologic abnormalities, although this effect is still being studied in humans. A small case series of 13 pregnancies in patients with aggressive MS with third trimester exposures to natalizumab displayed hematologic abnormalities in 10/13 births [62]. At this time, current prescribing information for natalizumab advises clinicians to weigh the benefits to the mother with aggressive disease and potential risks to the fetus when deciding whether or not to continue this DMT throughout pregnancy.

Overall, none of the DMTs used in MS is approved for use during pregnancy in the USA. FDA-approved DMTs not discussed above (e.g., daclizumab, alemtuzumab, ocrelizumab) have limited information available and no definitive conclusions as to their effects on the developing fetus. Each neurologist, in consultation with their patient, must seriously evaluate these medications' risk/benefit ratio with regard to patients who are interested in pregnancy. Ideally, this decision is made in consultation in a multidisciplinary manner with the patient's maternal/fetal/lactation specialists, if possible. Some general recommendations regarding the timeframes for stopping DMTs prior to conception and pregnancy are summarized in Table 3.

\section{Breastfeeding and MS}

One of the most common questions from pregnant patients with MS is how to navigate breastfeeding with respect to the use of disease-modifying medications. Informed decisions are needed regarding whether to breastfeed, for how long, and when to restart DMTs postpartum. The American Academy of Pediatrics currently recommends exclusive breastfeeding for infants for the first 6 months and then continued nursing after the introduction of solid foods for 1 year total or beyond when possible or desired by the mother. Theoretically, breastfeeding could affect the rate of postpartum relapses in the mother and/or impact the infant's development through medications that pass through breast milk to the infant. While there are no definitive answers, these issues warrant a review of information available to date.

\section{Effect of Breastfeeding on MS}

Several prospective studies and meta-analyses have looked at the effect of breastfeeding on postpartum MS relapses. No clear evidence exists that breastfeeding provides sufficient protection from relapses to warrant not restarting DMTs shortly after giving birth in women with active relapsing disease prior to pregnancy.

In the landmark study showing the significant reduction in relapse rates during the third trimester of pregnancy with rebound relapses postpartum, breastfeeding had no effect on postpartum relapses [10]. A follow-up analysis identified that the single best predictor of having a postpartum relapse was the pre-pregnancy relapse rate [64].

Another prospective study enrolled 201 women with MS, following them from pregnancy to 12 months postpartum and assessed the effect of breastfeeding on relapses. Women who breastfed were categorized into 2 subgroups: those who breastfed exclusively with no supplemental feedings for at least 2 months $(n=120)$ and those who breastfed partially but not exclusively $(n=81)$. In the first 6 months postpartum, the exclusive breastfeeding group had fewer relapses than women who partially breastfed or did not breastfeed at all [65]. However, the exclusive breastfeeding group also had 
Table 3 Suggested timeframes for stopping disease-modifying therapies prior to conception and pregnancy

\begin{tabular}{ll}
\hline FDA-approved MS therapy & $\begin{array}{l}\text { Number of recommended menstrual cycles to stop drug prior to } \\
\text { conception }\end{array}$ \\
\hline IFN- $\beta$ & $0-1$ \\
Glatiramer acetate & $0-1$ \\
Fingolimod [63] & 2 \\
Dimethyl fumarate & $0-1$ \\
Teriflunomide (prescribing & Two options because of long half-life (females and males): \\
$\quad$ information) & 1. Discontinue oral use 2 years in advance of conception \\
& 2. Perform accelerated elimination, use contraception until plasma \\
& 3 \\
Natalizumab [63] & $4-6$ \\
Alemtuzumab [63] (prescribing & \\
$\quad$ information) & 4 \\
Daclizumab [63] & 6 \\
Ocrelizumab (prescribing &
\end{tabular}

FDA = Food and Drug Administration; $\mathrm{MS}=$ multiple sclerosis; $\mathrm{IFN}=$ interferon fewer relapses during pregnancy and restarted DMTs in fewer numbers in the first 30 days postpartum. They also had used DMTs less frequently before conception than the nonexclusive group. Conversely, the nonexclusive breastfeeding group was more likely to be using DMTs at conception and to have a relapse during pregnancy. Thus, the nonexclusive breastfeeding group may have had more active MS which would likely be associated with restarting DMTs earlier. This hypothesis is consistent with authors of the PRIMS study, who concluded that the protective effects of breastfeeding may be confounded by the fact that patients with milder MS choose to breastfeed as compared with their counterparts with more active disease [64]. Additionally, a meta-analysis regarding breastfeeding and MS relapses also raised similar questions of whether or not the protective effect of breastfeeding on MS relapses was related to the confound of selection bias in the choice to breastfeed [66]. Mechanistically, it is unclear how breastfeeding would protect from relapses. Prolactin would continue to be high in those who are breastfeeding and prolactin has shown proinflammatory properties [67]. However, prolactin has shown neuroprotective properties related to remyelination [68], but this would not necessarily translate into an effect on relapse rates.

Overall, there are no clear answers as to when to restart DMTs and potentially interrupt or stop breastfeeding in patients with MS postpartum. Patients with more active disease prior to pregnancy may have more relapses postpartum and traditionally may be counseled to restart DMTs earlier. However, patients with milder MS may also have relapses postpartum. Individual patients and their clinicians must weigh their decision to breastfeed on a case-by-case basis as there is no conclusive evidence to support either choice.

\section{Breastfeeding and DMT Use}

To date, the FDA has generally recommended discontinuing DMTs while breastfeeding. Interestingly, this has not been based on scientific study but is more of a cautionary stance as there has not been enough concrete information on the excretion of the various MS medications into human breast milk or their effect on a developing infant. Some lactation studies have been undertaken recently looking at the relative infant dose (RID) received of DMTs when a mother with MS is nursing.

IFN- $\beta 1 \mathrm{a}$ is not generally excreted in breast milk owing to its large protein size and because it exists in a bound state with low plasma levels in the mother's body [69]. Additionally, beta IFNs are not orally bioavailable. This means that any small amount of beta IFNs present in breast milk are broken down and inactivated when ingested by an infant. In a study of 6 nursing mothers, the IFN- $\beta 1$ a calculated RID was only $0.006 \%$ of the maternal dose and no side effects were seen in the infants. Therefore, the authors concluded that it should be theoretically safe for a patient with MS to continue IFN- $\beta 1$ a while nursing. It is not known how applicable these data are to patients taking higher doses of beta IFNs, but given their similar molecular weight they most likely have similarly low levels in breast milk.

Natalizumab is excreted into breast milk, and the concentration of this medication therein appeared to increase by day 50 , as observed in a case report of a mother who started therapy while breastfeeding and had 2 doses of natalizumab at day 1 and day 29 [70]. The authors theorized that this increase in concentration may be due to the time it takes for natalizumab to reach a steady-state plasma level in the mother (about 
170 days). Although the calculated RID at day 50 was low $(5.3 \%)$, it may continue to rise with time and reach potentially toxic levels. Further, although IgG was previously thought to be broken down upon ingestion, there are receptors in the gut that may be used to transport $\operatorname{IgG}$ to plasma. Therefore, a monoclonal IgG4 antibody such as natalizumab may potentially be absorbed by an infant. Future studies are warranted before conclusions may be drawn; meanwhile, mothers with MS should discontinue breastfeeding while on natalizumab.

Mitoxantrone is a FDA-approved therapy for MS that is no longer commonly used as a DMT. It was studied in one mother who received 3 infusions. Although the calculated RID was between $2 \%$ and $12 \%$, because of the medication's high toxicity potential to the infant, it was deemed too risky to use while breastfeeding [71].

Newer FDA-approved therapies for MS have not yet been quantified in human breast milk and no RID has been calculated. Unfortunately, mouse and rat models are not useful in predicting human breast milk excretion and cannot be used as a predictor. However, as described above for other medications, one can infer the likelihood of whether or not a medication will be transferred into human breast milk based on the molecular weight, known protein binding, volume of distribution, lipid solubility, and if there are known active transport mechanisms for the drug [71]. Additionally, medications pass through the gut and liver of the infant and can be broken down via the first-pass effect. Finally, older infants are better able to metabolize than younger infants and may be able to tolerate medications better. Given the lack of data on breast milk transmission by DMTs and the fact that none is FDA approved for use during lactation, most patients will be counseled not to use DMTs at all while breastfeeding. However, by considering the probable transmission during lactation as summarized in Table 4 [71], patients and their clinicians can determine the best DMTs to take while breastfeeding if so desired. The authors of this paper recommend using caution in recommending nursing while using DMTs with considerable potential toxicity, even if it is unlikely to be transmitted into human breast milk.

\section{Management of Acute Relapses During Pregnancy and Breastfeeding}

In nonpregnant or non-nursing females, most MS relapses are treated with intravenous methylprednisolone or its oral equivalent. For MS relapses during pregnancy, intravenous SoluMedrol is considered safest to use during the second and third trimesters as Solu-Medrol is inactivated by the placenta. Steroids are generally avoided during the first trimester since they have been associated with craniofacial abnormalities. Intravenous immunoglobulin (IVIG), however, may be used safely throughout pregnancy and therefore may be preferable during the first trimester [72].
Table 4 Properties of multiple sclerosis disease-modifying therapies (DMT) with respect to transmission into breast milk and potential toxicity to the infant

\begin{tabular}{|c|c|c|}
\hline DMT & $\begin{array}{l}\text { Relevant medication } \\
\text { properties }\end{array}$ & $\begin{array}{l}\text { Transmission to human } \\
\text { breast milk (known or } \\
\text { theoretical) }\end{array}$ \\
\hline IFN- $\beta$ & Large MW & $\begin{array}{l}\text { Known transmission in } \\
\text { small amounts, RID } \\
0.006 \%\end{array}$ \\
\hline $\begin{array}{l}\text { Glatiramer } \\
\text { acetate }\end{array}$ & $\begin{array}{l}\text { Large MW, depolymerized } \\
\text { upon oral ingestion }\end{array}$ & $\begin{array}{l}\text { Unlikely transmission, no } \\
\text { data }\end{array}$ \\
\hline Natalizumab & $\begin{array}{l}\text { Prolonged time to reach } \\
\text { steady state ( } 28 \text { weeks) }\end{array}$ & $\begin{array}{l}\text { Known transmission in } \\
\text { human breast milk, RID } \\
2-5 \% \text { at } 50 \text { days before } \\
\text { drug reached steady } \\
\text { state, RID potentially } \\
\text { could increase with time }\end{array}$ \\
\hline Fingolimod & $\begin{array}{l}\text { Very protein bound, high } \\
\text { oral bioavailability }\end{array}$ & $\begin{array}{l}\text { Likely small amounts } \\
\text { would be transmitted, } \\
\text { would be orally available } \\
\text { to infant }\end{array}$ \\
\hline $\begin{array}{l}\text { Dimethyl } \\
\text { fumarate }\end{array}$ & $\begin{array}{l}\text { Low MW, high volume of } \\
\text { distribution, short } \\
\text { half-life }\end{array}$ & Likely transmission, no data \\
\hline Teriflunomide & $\begin{array}{l}\text { Long half-life, very protein } \\
\text { bound }\end{array}$ & Likely transmission, no data \\
\hline Rituximab & Large MW, long half-life & $\begin{array}{l}\text { Unlikely transmission, no } \\
\text { data }\end{array}$ \\
\hline Daclizumab & $\begin{array}{l}\text { Low volume of } \\
\text { distribution, MW of } 144 \\
\mathrm{kD} \text { limits ability to pass } \\
\text { into breast milk }\end{array}$ & $\begin{array}{l}\text { Transmission likely limited, } \\
\text { no data }\end{array}$ \\
\hline Alemtuzumab & Large MW & $\begin{array}{l}\text { Unlikely transmission in } \\
\text { significant amounts, no } \\
\text { data. }\end{array}$ \\
\hline
\end{tabular}

Revised from [71]

$\mathrm{MW}=$ molecular weight; $\mathrm{RID}=$ relative infant dose (compared with maternal dose)

Traditionally, it was recommended that a nursing mother stop breastfeeding when taking steroids as they were known to be excreted into breast milk [72]. However, until recently this amount of excretion had not been quantified. One mother with MS undergoing treatment with intravenous methylprednisolone once a day for 3 days submitted breast-milk samples for analysis. Her infant did not breastfeed during this timeframe. Breast-milk concentrations of methylprednisolone were highest at $1 \mathrm{~h}$ after administration and quickly tapered off. Another study determined that even though the intravenous steroid dose delivered to the mother was quite high, the RID that would be ingested by an infant was only between $1.1 \%$ and $1.5 \%$, well below the generally accepted limit RID of $10 \%$ for medications excreted into breast milk [73]. It was also below the calculated dose of methylprednisolone on a $\mathrm{mg} /$ $\mathrm{kg} /$ day basis for infants who require steroid treatment for pediatric medical conditions. Nevertheless, the authors 
concluded that if a mother with MS desired to continue breastfeeding while being treated with a short course of IV methylprednisolone, she could consider doing so 2 to $4 \mathrm{~h}$ after each of her intravenous doses.

IVIG is also used for the treatment of MS relapses and has long been considered safe to prescribe to a lactating mother, based upon a retrospective study of 28 patients treated throughout pregnancy and postpartum with IVIG where no adverse effects were observed in the fetus, infant, or mother. For this reason, IVIG has often been the preferred medication when treating MS relapses in the pregnant and/or nursing mother [72].

Another consideration when a relapse arises in a pregnant or nursing mother is the potential need for further testing (e.g., MRI scanning) to assess for new lesions. The use of MRI is considered safe during pregnancy. Traditionally, it has been recommended that MRI is avoided during the first trimester owing to its potential to heat developing tissues, but a recent analysis has not shown increased risk to a fetus for noncontrast MRI. The use of the contrast agent gadolinium, however, is strongly discouraged during any trimester of pregnancy unless its benefits to the mother outweigh the risks since it may have a multitude of negative effects on the developing fetus and early childhood, including rheumatologic disorders, skin and inflammatory conditions, and neonatal death [74]. All other common tests used in patients with MS, such as evoked potentials and lumbar punctures, are safe for use during pregnancy.

Gadolinium contrast is excreted in breast milk in very small amounts and the overall amount transferred to the infant orally is $<1 \%$ of the intravenous gadolinium dosage a newborn would receive for a radiologic procedure [75]. Additionally, only a fraction of that fraction of contrast would actually be absorbed from the infant's gut, calculated to be roughly $0.0004 \%$ of the mother's intravenous dose [76]. According to the American College of Obstetrics and Gynecologists' guidelines, patients can either continue breastfeeding or, if they wish to avoid any ingestion at all by the infant, they can "pump and dump" for $24 \mathrm{~h}$ after gadolinium contrast exposure.

When comparisons are made between gadolinium exposure levels in the breastfeeding infant to exposure levels in an infant undergoing an imaging procedure by the pediatrician, it is important to appreciate that the risk:benefit ratio is very different. In the former, risk of gadolinium is weighed versus breastfeeding of a healthy infant, while in the latter, risk of gadolinium is weighed against the need for a medically indicated intervention in the infant. Minimal to no risk is acceptable in the former, whereas some risk is acceptable in the latter.

\section{Overall Conclusions}

Pregnancy is clearly a major disease modifier in MS with relative protection from relapses during the third trimester and rebound relapses postpartum. Whether being multiparous has long-term beneficial effects on disease susceptibility or disability remains unknown. Clinical trials have aimed to simulate pregnancy's beneficial effect. Two phase II trials have been promising for estriol, with a third trial ongoing (NCT01466114). In a trial using estradiol, there was a trend of benefit that was dose dependent, while a trial using progesterone treatment was negative. The use of MS treatments to manage disease requires the clinician and patient to work closely in planning for an optimal outcome for both the patient and child. Key factors to consider are the timing of conception in relation to MS medication use, treatment of relapses before, during and after pregnancy, and balancing the desire to breastfeed with the need for MS medication. By outlining options in advance, patients and their healthcare team can together manage disease while reducing the risk of exposure to medications by the developing fetus or breastfeeding infant.

Acknowledgements This work was funded by National Institutes of Health grants R01NS096748, the Conrad N. Hilton Foundation grant \#20150232, the California Community Foundation \#BAPP-15-118094, each to R.V., as well as by the Tom Sherak MS Hope Foundation.

Required Author Forms Disclosure forms provided by the authors are available with the online version of this article.

\section{References}

1. Voskuhl RR, Gold SM. Sex-related factors in multiple sclerosis susceptibility and progression. Nat Rev Neurol 2012;8(5):255-263.

2. Whitacre CC, Reingold SC, O'Looney PA. A gender gap in autoimmunity. Science 1999;283(5406):1277-1278.

3. Gold SM, Voskuhl RR. Pregnancy and multiple sclerosis: from molecular mechanisms to clinical application. Semin Immunopathol 2016;38(6):709-718.

4. Arevalo MA, Santos-Galindo M, Bellini MJ, Azcoitia I, GarciaSegura LM. Actions of estrogens on glial cells: implications for neuroprotection. Biochim Biophys Acta 2010;1800(10):11061112.

5. Brann DW, Dhandapani K, Wakade C, Mahesh VB, Khan MM. Neurotrophic and neuroprotective actions of estrogen: Basic mechanisms and clinical implications. Steroids 2007;72(5):381-405.

6. Nilsen J, Diaz Brinton R. Mechanism of estrogen-mediated neuroprotection: regulation of mitochondrial calcium and $\mathrm{Bcl}-2$ expression. Proc Natl Acad Sci U S A 2003;100(5):2842-2847.

7. Spence RD, Voskuhl RR. Neuroprotective effects of estrogens and androgens in CNS inflammation and neurodegeneration. Front Neuroendocrinol 2012;33(1):105-115.

8. Babayan AH, Kramar EA. Rapid effects of oestrogen on synaptic plasticity: interactions with actin and its signalling proteins. $\mathrm{J}$ Neuroendocrinol 2013;25(11):1163-1172.

9. Liu F, Day M, Muniz LC, et al. Activation of estrogen receptor-beta regulates hippocampal synaptic plasticity and improves memory. Nat Neurosci 2008;11(3):334-343.

10. Confavreux C, Hutchinson M, Hours MM, Cortinovis-Tourniaire P, Moreau T. Rate of pregnancy-related relapse in multiple sclerosis. Pregnancy in Multiple Sclerosis Group. N Engl J Med 1998;339(5): 285-291.

11. D'Hooghe M B, De Keyser J. Multiparity in women with multiple sclerosis causes less long-term disability: yes. Mult Scler 2014;20(11):1432-1434. 
12. Hutchinson M. Multiparity in women with multiple sclerosis causes less long-term disability: commentary. Mult Scler 2014;20(11): 1437-1438.

13. McCombe PA, Callaway LK. Multiparity in women with multiple sclerosis causes less long-term disability: no. Mult Scler 2014;20(11):1435-1436.

14. D'Hooghe M B, Haentjens P, Nagels G, D'Hooghe T, De Keyser J. Menarche, oral contraceptives, pregnancy and progression of disability in relapsing onset and progressive onset multiple sclerosis. $\mathrm{J}$ Neurol 2012;259(5):855-861.

15. Masera S, Cavalla P, Prosperini L, et al. Parity is associated with a longer time to reach irreversible disability milestones in women with multiple sclerosis. Mult Scler 2015;21(10):1291-1297.

16. Runmarker B, Andersen O. Pregnancy is associated with a lower risk of onset and a better prognosis in multiple sclerosis. Brain 1995;118 (Pt 1):253-261.

17. Jokubaitis VG, Spelman T, Kalincik T, et al. Predictors of long-term disability accrual in relapse-onset multiple sclerosis. Ann Neurol 2016;80(1):89-100.

18. Ponsonby AL, Lucas RM, van der Mei IA, et al. Offspring number, pregnancy, and risk of a first clinical demyelinating event: the AusImmune Study. Neurology 2012;78(12):867-874.

19. Orton SM, Herrera BM, Yee IM, et al. Sex ratio of multiple sclerosis in Canada: a longitudinal study. Lancet Neurol 2006;5(11):932-936.

20. Daumer M, Weinshenker BG, Voskuhl R. Pregnancy: a "modifiable" risk factor in MS? Neurology 2012;78(12):846-848.

21. Bebo BF, Jr., Fyfe-Johnson A, Adlard K, Beam AG, Vandenbark AA, Offner H. Low-dose estrogen therapy ameliorates experimental autoimmune encephalomyelitis in two different inbred mouse strains. J Immunol 2001;166(3):2080-2089.

22. Jansson L, Olsson T, Holmdahl R. Estrogen induces a potent suppression of experimental autoimmune encephalomyelitis and collagen-induced arthritis in mice. J Neuroimmunol 1994;53(2): 203-207.

23. Kim S, Liva SM, Dalal MA, Verity MA, Voskuhl RR. Estriol ameliorates autoimmune demyelinating disease: implications for multiple sclerosis. Neurology 1999;52(6):1230-1238.

24. Palaszynski KM, Liu H, Loo KK, Voskuhl RR. Estriol treatment ameliorates disease in males with experimental autoimmune encephalomyelitis: implications for multiple sclerosis. J Neuroimmunol 2004;149(1-2):84-89.

25. Papenfuss TL, Powell ND, McClain MA, et al. Estriol generates tolerogenic dendritic cells in vivo that protect against autoimmunity. J Immunol 2011;186(6):3346-3355.

26. Ziehn MO, Avedisian AA, Dervin SM, O'Dell TJ, Voskuhl RR. Estriol preserves synaptic transmission in the hippocampus during autoimmune demyelinating disease. Lab Invest 2012;92(8):1234-1245

27. Head KA. Estriol: safety and efficacy. Altern Med Rev 1998;3(2): 101-113.

28. Lauritzen C. Results of a 5 years prospective study of estriol succinate treatment in patients with climacteric complaints. Hormone Metab Res 1987;19(11):579-584.

29. Granberg S, Eurenius K, Lindgren R, Wilhelmsson L. The effects of oral estriol on the endometrium in postmenopausal women. Maturitas 2002;42(2):149-156.

30. Taylor M. Unconventional estrogens: estriol, biest, and triest. Clin Obstet Gynecol 2001;44(4):864-879.

31. Sicotte NL, Liva SM, Klutch R, et al. Treatment of multiple sclerosis with the pregnancy hormone estriol. Ann Neurol 2002;52(4): 421-428.

32. Gold SM, Sasidhar MV, Morales LB, et al. Estrogen treatment decreases matrix metalloproteinase (MMP)-9 in autoimmune demyelinating disease through estrogen receptor alpha (ERalpha). Lab Invest 2009;89(10):1076-1083.
33. Soldan SS, Retuerto AI, Sicotte NL, Voskuhl RR. Immune modulation in multiple sclerosis patients treated with the pregnancy hormone estriol. J Immunol 2003;171(11):6267-6274.

34. Voskuhl RR, Wang H, Wu TC, et al. Estriol combined with glatiramer acetate for women with relapsing-remitting multiple sclerosis: a randomised, placebo-controlled, phase 2 trial. Lancet Neurol 2016;15(1):35-46.

35. Voskuhl R, Wang H, Elashoff RM. Why use sex hormones in relapsing-remitting multiple sclerosis? Authors' reply. Lancet Neurol 2016;15(8):790-791.

36. Pozzilli C, De Giglio L, Barletta VT, et al. Oral contraceptives combined with interferon beta in multiple sclerosis. Neurol Neuroimmunol Neuroinflamm 2015;2(4):e120.

37. De Giglio L, Marinelli F, Barletta VT, et al. Effect on cognition of estroprogestins combined with interferon beta in multiple sclerosis: analysis of secondary outcomes from a randomised controlled trial. CNS Drugs 2017;31(2):161-168.

38. Vukusic S, Ionescu I, El-Etr M, et al. The Prevention of PostPartum Relapses with Progestin and Estradiol in Multiple Sclerosis (POPART'MUS) trial: rationale, objectives and state of advancement. J Neurol Sci 2009;286(1-2):114-118.

39. Gago N, Akwa Y, Sananes N, et al. Progesterone and the oligodendroglial lineage: stage-dependent biosynthesis and metabolism. Glia 2001;36(3):295-308.

40. Schumacher M, Guennoun R, Stein DG, De Nicola AF. Progesterone: therapeutic opportunities for neuroprotection and myelin repair. Pharmacol Ther 2007;116(1):77-106.

41. Giatti S, Caruso D, Boraso M, et al. Neuroprotective effects of progesterone in chronic experimental autoimmune encephalomyelitis. J Neuroendocrinol 2012;24(6):851-861.

42. Hoffman GE, Le WW, Murphy AZ, Koski CL. Divergent effects of ovarian steroids on neuronal survival during experimental allergic encephalitis in Lewis rats. Exp Neurol 2001;171(2):272-284.

43. Jansson L, Holmdahl R. Oestrogen induced suppression of collagen arthritis. IV: Progesterone alone does not affect the course of arthritis but enhances the oestrogen-mediated therapeutic effect. J Reprod Immunol 1989;15(2):141-150.

44. Voskuhl RR, Palaszynski KM. Female sex hormones at supraphysiologic, but not physiologic, levels decrease EAE severity in female SJL mice. FASEB J 2001;15(4):A372.

45. Correale J, Ysrraelit MC, Gaitan MI. Gender differences in 1,25 dihydroxyvitamin D3 immunomodulatory effects in multiple sclerosis patients and healthy subjects. J Immunol 2010;185(8):4948-4958.

46. Cutolo M, Paolino S, Sulli A, Smith V, Pizzorni C, Seriolo B. Vitamin D, steroid hormones, and autoimmunity. Ann N Y Acad Sci 2014;1317:39-46.

47. Disanto G, Handel AE, Ramagopalan SV. Estrogen-vitamin D interaction in multiple sclerosis. Fertil Steril 2011;95(1):e3.

48. Rolf L, Damoiseaux J, Hupperts R, Huitinga I, Smolders J. Network of nuclear receptor ligands in multiple sclerosis: common pathways and interactions of sex-steroids, corticosteroids and vitamin D3-derived molecules. Autoimmun Rev 2016;15:900-910.

49. Spanier JA, Nashold FE, Mayne CG, Nelson CD, Hayes CE. Vitamin D and estrogen synergy in Vdr-expressing CD4(+) T cells is essential to induce Helios(+)FoxP3(+) T cells and prevent autoimmune demyelinating disease. J Neuroimmunol 2015;286:48-58.

50. Engler JB, Kursawe N, Solano ME, et al. Glucocorticoid receptor in $\mathrm{T}$ cells mediates protection from autoimmunity in pregnancy. Proc Natl Acad Sci U S A 2017;114(2):E181-E190.

51. Correale J, Farez MF, Ysrraelit MC. Increase in multiple sclerosis activity after assisted reproduction technology. Ann Neurol 2012;72(5):682-694.

52. Finer LB, Zolna MR. Unintended pregnancy in the United States: incidence and disparities, 2006. Contraception 2011;84(5):478-485. 
53. Alonso A, Jick SS, Olek MJ, Ascherio A, Jick H, Hernan MA. Recent use of oral contraceptives and the risk of multiple sclerosis. Arch Neurol 2005;62(9):1362-135.

54. Hernan MA, Hohol MJ, Olek MJ, Spiegelman D, Ascherio A. Oral contraceptives and the incidence of multiple sclerosis. Neurology 2000;55(6):848-854.

55. Thorogood M, Hannaford PC. The influence of oral contraceptives on the risk of multiple sclerosis. Br J Obstet Gynaecol 1998;105(12):1296-1299.

56. Houtchens MK, Kolb CM. Multiple sclerosis and pregnancy: therapeutic considerations. J Neurol 2013;260(5):1202-1214.

57. Thiel S, Langer-Gould A, Rockhoff M, et al. Interferon-beta exposure during first trimester is safe in women with multiple sclerosis-a prospective cohort study from the German Multiple Sclerosis and Pregnancy Registry. Mult Scler 2016;22(6):801-809.

58. Coyle PK, Sinclair SM, Scheuerle AE, Thorp JM, Jr., Albano JD, Rametta MJ. Final results from the Betaseron (interferon beta-1b) Pregnancy Registry: a prospective observational study of birth defects and pregnancy-related adverse events. BMJ Open 2014;4(5): e004536.

59. Karlsson G, Francis G, Koren G, et al. Pregnancy outcomes in the clinical development program of fingolimod in multiple sclerosis. Neurology 2014;82(8):674-680

60. Gold R, Phillips JT, Havrdova E, et al. Delayed-release dimethyl fumarate and pregnancy: preclinical studies and pregnancy outcomes from clinical trials and postmarketing experience. Neurol Ther 2015;4(2):93-104.

61. Kieseier BC, Benamor M. Pregnancy outcomes following maternal and paternal exposure to teriflunomide during treatment for relapsing-remitting multiple sclerosis. Neurol Ther 2014;3(2):133-138.

62. Friend S, Richman S, Bloomgren G, Cristiano LM, Wenten M. Evaluation of pregnancy outcomes from the Tysabri(R) (natalizumab) pregnancy exposure registry: a global, observational, follow-up study. BMC Neurol 2016;16(1):150.

63. Cree BA. Update on reproductive safety of current and emerging disease-modifying therapies for multiple sclerosis. Mult Scler 2013;19(7):835-843.
64. Vukusic S, Hutchinson M, Hours M, et al. Pregnancy and multiple sclerosis (the PRIMS study): clinical predictors of post-partum relapse. Brain 2004;127(Pt 6):1353-1360.

65. Hellwig K, Rockhoff M, Herbstritt S, et al. Exclusive Breastfeeding and the Effect on Postpartum Multiple Sclerosis Relapses. JAMA Neurol 2015;72(10):1132-1138.

66. Pakpoor J, Disanto G, Lacey MV, Hellwig K, Giovannoni G, Ramagopalan SV. Breastfeeding and multiple sclerosis relapses: a meta-analysis. J Neurol 2012;259(10):2246-2248.

67. Riskind PN, Massacesi L, Doolittle TH, Hauser SL. The role of prolactin in autoimmune demyelination: suppression of experimental allergic encephalomyelitis by bromocriptine. Ann Neurol 1991;29(5):542-547.

68. Gregg C, Shikar V, Larsen P, et al. White matter plasticity and enhanced remyelination in the maternal CNS. J Neurosci 2007;27(8):1812-1823.

69. Hale TW, Siddiqui AA, Baker TE. Transfer of interferon beta-1a into human breastmilk. Breastfeed Med 2012;7(2):123-125.

70. Baker TE, Cooper SD, Kessler L, Hale TW. Transfer of natalizumab into breast milk in a mother with multiple sclerosis. J Hum Lact 2015;31(2):233-236.

71. Almas S, Vance J, Baker T, Hale T. Management of multiple sclerosis in the breastfeeding mother. Mult Scler Int 2016;2016: 6527458.

72. Argyriou AA, Makris N. Multiple sclerosis and reproductive risks in women. Reprod Sci 2008;15(8):755-764.

73. Cooper SD, Felkins K, Baker TE, Hale TW. Transfer of methylprednisolone into breast milk in a mother with multiple sclerosis. J Hum Lact 2015;31(2):237-239.

74. Ray JG, Vermeulen MJ, Bharatha A, Montanera WJ, Park AL. Association between MRI exposure during pregnancy and fetal and childhood outcomes. JAMA 2016;316(9):952-961.

75. Sundgren PC, Leander P. Is administration of gadolinium-based contrast media to pregnant women and small children justified? J Magn Reson Imaging 2011;34(4):750-757.

76. Tirada N, Dreizin D, Khati NJ, Akin EA, Zeman RK. Imaging pregnant and lactating patients. Radiographics 2015;35(6):17511765. 